\section{Neutrophils and tissue damage: is hypoxia the key to excessive degranulation?}

\author{
Andrew E Williams, Rachel C Chambers
}

Neutrophils are the first innate immune cells recruited into sites of infection and inflammation, and although they are critical for host defence against microbial pathogens, they have also been associated with local tissue damage. Predominant neutrophilic inflammation is a key hallmark of many respiratory diseases, and the release of proteinases and reactive oxygen species (ROS) from primed neutrophils has been implicated in mediating the damage to surrounding tissue. ${ }^{1}$ Inflammation is further accompanied by severe hypoxia (lack of oxygen) within the affected tissue, and is a characteristic of both acute and chronic lung diseases, such as acute respiratory distress syndrome (ARDS), ${ }^{2}$ COPD $^{3}$ and cystic fibrosis (CF). ${ }^{4}$ Considering that tissue hypoxia is part of a normal inflammatory response, innate immune cells such as neutrophils and macrophages possess important cellular and molecular mechanisms that enable them to function effectively at low oxygen concentrations. ${ }^{5}$

There is considerable interplay between the molecular pathways that regulate hypoxia and inflammation. In previous studies, mice exposed to hypoxic conditions $\left(5 \% \mathrm{O}_{2}\right.$ for 1 hour $)$ demonstrated increased levels of interleukin 6 (IL-6), tumour necrosis factor and IL-1 in both serum and isolated macrophages. ${ }^{6}$ In humans, increased levels of IL-6, IL-1RA and C-reactive protein were detected in serum after volunteers experienced three overnight stays at altitude. ${ }^{7}$ Moreover, infiltrating neutrophils directly increase their oxygen consumption in order to assemble the nicotinamide adenine dinucleotide phosphate oxidase complex that generates ROS and, in conjunction with superoxide dismutase and myeloperoxidase (MPO), hydrogen peroxide $\left(\mathrm{H}_{2} \mathrm{O}_{2}\right)$ and hypochlorous acid $(\mathrm{HClO})$, thereby depleting local levels of

Division of Medicine, Centre for Inflammation and Tissue Repair, UCL Respiratory, University College London, London, UK

Correspondence to Professor Rachel C Chambers, Centre for Inflammation and Tissue Repair, Division of Medicine, University College London, London WC1E 6JF, UK; r.chambers@ucl.ac.uk oxygen. ${ }^{8}{ }^{9}$ Neutrophils are also thought to influence the tissue microenvironment during episodes of hypoxia. For example, neutrophil migration across epithelia increases the transcriptional activity of hypoxia-inducible genes in epithelial cells, which in turn influence the resolution of inflammation. ${ }^{10}$

Neutrophils have, therefore, been implicated in the pathogenesis of several inflammatory lung diseases associated with tissue hypoxia. ${ }^{5}$ However, the mechanisms by which neutrophils mediate tissue damage under hypoxic conditions are less clear. Under homeostatic conditions neutrophils are short-lived cells that readily undergo apoptosis in order to avoid the release of toxic granule contents. However, under hypoxic conditions neutrophil survival is prolonged, which is thought to delay the resolution of inflammation and promote tissue damage, potentially through the release of destructive proteinases or ROS. ${ }^{11}$ The study by Hoenderdos et al in this issue of Thorax aims to explore the functional link between hypoxia and the regulation of neutrophil degranulation in the context airway inflammation.

Compared with neutrophils incubated under normoxic conditions (atmospheric $\mathrm{O}_{2}$ concentration), this study demonstrated that neutrophils incubated under hypoxic conditions $\left(0.8 \% \mathrm{O}_{2}, 3 \mathrm{kPa}\right.$ for 4 hours $)$ released significantly elevated levels of neutrophil elastase (NE), MPO, lactoferrin and matrix metalloproteinase-9, thereby indicating degranulation of azurophil (primary), specific (secondary) and gelatinase (tertiary) granules. This effect was largely dependent on priming with granulocyte-macrophage colony-stimulating factor (GM-CSF) and activation with formylated peptide (fMLP), as little degranulation occurred at basal levels in either normoxic or hypoxic conditions. These findings support previous studies demonstrating that neutrophils isolated from human volunteers, following a period of hypoxaemia, released increased levels of $\mathrm{NE}$ after fMLP stimulation, ${ }^{12}$ and neutrophils from healthy volunteers exposed to hypoxia ex vivo (3 kPA for 1-4 hours) released NE. ${ }^{13}$ However, these two studies reported opposing effects on ROS production.
Previous studies under hypoxia have sometimes given conflicting results, which is likely related to differing experimental conditions that affect neutrophil function. There may, therefore, be some merit in standardising methodologies across laboratories, to enable direct comparison of studies within the scientific community. It is also important to consider whether experimental hypoxic conditions mirror those found in the lung during episodes of inflammation. The lung is normally an oxygen-rich organ, with an alveolar $\mathrm{PO}_{2}$ $\sim 110 \mathrm{~mm} \mathrm{Hg}$ compared with other systemic organs $(3-20 \mathrm{~mm} \mathrm{Hg})$. Therefore, oxygen tensions in a hypoxic lung are higher than other organs $(\sim 50 \mathrm{~mm} \mathrm{Hg}$ compared with less than $10 \mathrm{~mm} \mathrm{Hg}){ }^{14}$ The current study exposed neutrophils to hypoxic conditions of $3 \mathrm{kPa}$, which is equivalent to $\sim 20 \mathrm{~mm} \mathrm{Hg}$, and therefore attempts to recapitulate the physiological conditions within the airway epithelium. It is argued that similar mechanistic ex vivo studies should also reflect these unique conditions. More challenging will be to recapitulate hypoxic conditions within tissue niches or foci of inflammation, such as those that occur in the small airway epithelium in COPD and CF.

The release of neutrophil proteinases has been strongly implicated in promoting tissue damage within inflamed lung. ${ }^{15}$ In this study, hypoxic supernatants from GM-CSF-stimulated and fMLP-stimulated neutrophils increased the detachment and death of epithelial cells compared with normoxic neutrophils. Treatment with $\alpha-1$-anti-trypsin further revealed that the toxic effects were mediated by proteinases, supporting a role for activated neutrophils in mediating tissue damage. The enhanced toxic effects of hypoxic neutrophil supernatants were apparent when epithelial cells were cultured in twodimensional (2D) monolayers. However, recapitulating these findings in $3 \mathrm{D}$ culture systems or, more importantly in vivo, remain a considerable challenge.

It is important to fully appreciate the significant effect that hypoxia has on downstream signalling pathways in both tissue-resident cells and infiltrating leukocytes. Eukaryotic cells have evolved several oxygen-sensing pathways, of which the principal regulators are hypoxia-inducible transcription factor-1 and transcription factor-2 (HIF-1 and HIF-2). ${ }^{16}$ Under hypoxic conditions HIFs are stabilised, allowing them to translocate to the nucleus and regulate HIF response genes. ${ }^{17}$ Interestingly, the current study reports that hypoxia-induced degranulation of neutrophils is not primarily 
mediated by HIF-1. Treatment of neutrophils with either dimethyloxalyl glycine or desferrioxamine, which are both HIF-1-stabilising agents, did not affect NE release. Furthermore, hypoxia did not increase the expression of $\mathrm{NE}$ but $\mathrm{did}$ increase the expression of BCL2/ Adenovirus E1B $19 \mathrm{kDa}$ Interacting Protein 2, both of which are putative HIF-1 response genes. Taken together, these data suggest that NE release following hypoxia-induced degranulation may be HIF-1 independent, although the contribution of HIF-2 was not directly explored in this study.

Neutrophil degranulation is also thought to be dependent on actin cytoskeletal rearrangements. Indeed, hypoxia caused focal actin polymerisation in fMLP-stimulated neutrophils. However, inhibition of actin polymerisation using cytochalasin B increased NE release, while treatment with jasplakinolide (which promotes actin polymerisation) had no significant effect on NE release. Actin remodelling is necessary for neutrophil degranulation, as well as polarisation and migration in response to exogenous stimuli such as fMLP and IL-8. ${ }^{18}$ Cytoskeletal rearrangements are thought to be a prerequisite for the intracellular movement of granules within the cytoplasm, while actin disassembly is necessary for access to the inner surface of the plasma membrane. It is, therefore, plausible that cytochalasin B relaxes the actin cytoskeleton and thereby promotes granule fusion with the plasma membrane, in a similar way that immune synapses form during polarised secretion of cytotoxic T-cell granules. ${ }^{19}$

Degranulation is a tightly regulated process involving the activation of G-protein-coupled receptors, intracellular calcium mobilisation and ATP-dependent phosphorylation of downstream signalling molecules. ${ }^{18}$ Treatment with a phospholipase $\mathrm{C}$ inhibitor or ethylene glycol tetraacetic acid, in order to block intracellular $\mathrm{Ca}^{2+}$, inhibited NE release under hypoxic conditions. Furthermore, treatment with thapsigargin, which is a $\mathrm{Ca}^{2+}$ ATPase inhibitor that elevates intracellular $\mathrm{Ca}^{2+}$ levels, caused an increase in NE release. These studies, therefore, confirm that $\mathrm{Ca}^{2+}$ mobilisation is an important signalling event during neutrophil degranulation under hypoxic conditions. However, the specific downstream targets of $\mathrm{Ca}^{2+}$ during neutrophil degranulation remain largely unknown.

Considering that hypoxia-induced neutrophil degranulation was independent of
HIF signalling, an alternative phosphoinositide 3-kinase (PI3K) signalling pathway was investigated. A pan PI3K inhibitor reduced neutrophil survival under hypoxic conditions, while a pan PI3K inhibitor and a PI3K $\gamma$ inhibitor completely abolished NE release from GM-CSFstimulated and fMLP-stimulated neutrophils. Inhibition of PI3K $\gamma$ also inhibited the downstream phosphorylation of Akt, suggesting that the PI3K-Akt pathway may play a role in hypoxia-mediated neutrophil degranulation. It will be interesting to explore whether other known regulators of neutrophil degranulation, such as Src family kinases, guanosine triphosphatases, such as Ras and Rho, and MAP kinases, are also regulated by hypoxia. In addition, hypoxia, PI3K/Akt and nuclear factor- $\kappa \mathrm{B}$ $(\mathrm{NF}-\kappa \mathrm{B})$ pathways interact to promote proinflammatory and HIF-target gene expression. Although not explored in the current study, the interplay between hypoxia-mediated signalling pathways and NF- $\kappa$ B-mediated pathways is likely to be functionally important in driving neutrophilic inflammation. ${ }^{11}$

In summary, the current study by Hoenderdos et al in this issue of Thorax highlights the importance of hypoxia in regulating neutrophil degranulation. The continued release of neutrophil-derived proteinases is likely to contribute to the tissue damage observed in acute and chronic lung diseases, such as ARDS, CF and COPD. Studying neutrophil function in hypoxic conditions ex vivo may therefore reveal novel, drug-targetable cellular and molecular processes. The future challenge will be to reproduce the physiological conditions that occur within an injured lung as closely as possible.

Twitter Follow Andrew Williams and CITR at @AndrewEWilliams and @LungLab

Contributors AEW and RCC wrote and edited the manuscript.

Competing interests None declared.

Provenance and peer review Commissioned; externally peer reviewed.

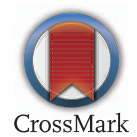

To cite Williams AE, Chambers RC. Thorax 2016;71:977-978.

Published Online First 1 September 2016

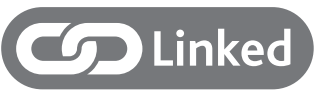

http://dx.doi.org/10.1136/thoraxjnl-2015-207604
Thorax 2016:71:977-978.

doi:10.1136/thoraxjnl-2016-208879

\section{REFERENCES}

1 Worthen GS, Haslett C, Rees AJ, et al. Neutrophil-mediated pulmonary vascular injury. Synergistic effect of trace amounts of lipopolysaccharide and neutrophil stimuli on vascular permeability and neutrophil sequestration in the lung. Am Rev Respir Dis 1987;136:19-28.

2 Han S, Mallampalli RK. The acute respiratory distress syndrome: from mechanism to translation. J Immunol 2015;194:855-60.

3 Hoenderdos K, Condliffe A. The neutrophil in chronic obstructive pulmonary disease. Am J Respir Cell Mol Biol 2013;48:531-9.

4 lannitti RG, Casagrande A, De Luca LA, et al. Hypoxia promotes danger-mediated inflammation via receptor for advanced glycation end products in cystic fibrosis. Am J Respir Crit Care Med 2013;188:1338-50.

5 Thompson AA, Binham J, Plant T, et al. Hypoxia, the HIF pathway and neutrophilic inflammatory responses. Biol Chem 2013;394:471-7.

6 Ertel W, Morrison MH, Ayala A, et al. Hypoxemia in the absence of blood loss or significant hypotension causes inflammatory cytokine release. Am J Physiol 1995;269(Pt 2):R160-6.

7 Hartmann G, Tschöp M, Fischer R, et al. High altitude increases circulating interleukin-6, interleukin-1 receptor antagonist and C-reactive protein. Cytokine 2000;12:246-52.

8 Rosen H, Crowley JR, Heinecke JW. Human neutrophils use the myeloperoxidase-hydrogen peroxide-chloride system to chlorinate but not nitrate bacterial proteins during phagocytosis. J Biol Chem 2002;277:30463-8.

9 Egners A, Erdem M, Cramer T. The response of macrophages and neutrophils to hypoxia in the context of cancer and other inflammatory diseases. Mediators Inflamm 2016;2016:2053646.

10 Campbell EL, Bruyninckx WJ, Kelly CJ, et al. Transmigrating neutrophils shape the mucosal microenvironment through localized oxygen depletion to influence resolution of inflammation. Immunity 2014;40:66-77.

11 Walmsley SR, Print C, Farahi N, et al. Hypoxia-induced neutrophil survival is mediated by HIF-1alpha-dependent NF-kappaB activity. J Exp Med 2005;201:105-15.

12 Tamura DY, Moore EE, Partrick DA, et al. Acute hypoxemia in humans enhances the neutrophil inflammatory response. Shock 2002;17:269-73.

13 McGovern NN, Cowburn AS, Porter L, et al. Hypoxia selectively inhibits respiratory burst activity and killing of Staphylococcus aureus in human neutrophils. J Immunol 2011;186:453-63.

14 Fröhlich S, Boylan J, McLoughlin P. Hypoxia-induced inflammation in the lung: a potential therapeutic target in acute lung injury? Am J Respir Cell Mol Biol 2013;48:271-9.

15 Zemans RL, Colgan SP, Downey GP. Transepithelial migration of neutrophils: mechanisms and implications for acute lung injury. Am J Respir Cell Mol Biol 2009;40:519-35.

16 Walmsley SR, McGovern NN, Whyte MK, et al. The HIF/VHL pathway: from oxygen sensing to innate immunity. Am J Respir Cell Mol Biol 2008;38:251-5.

17 Semenza GL. Oxygen sensing, hypoxia-inducible factors, and disease pathophysiology. Annu Rev Pathol 2014;9:47-71.

18 Lacy P. Mechanisms of degranulation in neutrophils. Allergy Asthma Clin Immunol 2006;2:98-108.

19 Ritter AT, Asano Y, Stinchcombe JC, et al. Actin depletion initiates events leading to granule secretion at the immunological synapse. Immunity 2015;42:864-76. 